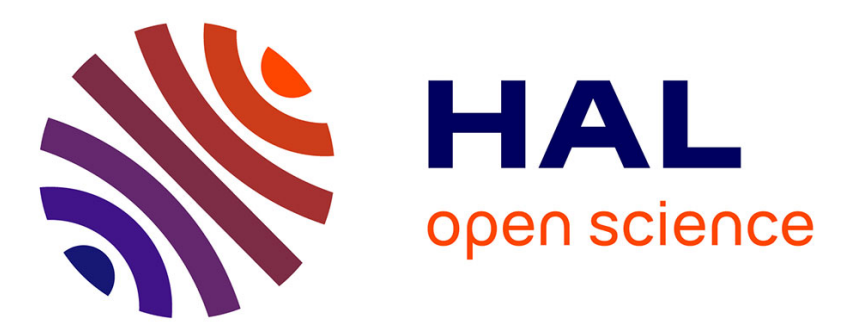

\title{
Drosophila ammonium transporter Rh50 is required for integrity of larval muscles and neuromuscular system
}

\author{
Mathilde Lecompte, Daniel Cattaert, Alain Vincent, Serge Birman, Baya
}

Chérif-zahar

\section{- To cite this version:}

Mathilde Lecompte, Daniel Cattaert, Alain Vincent, Serge Birman, Baya Chérif-zahar. Drosophila ammonium transporter Rh50 is required for integrity of larval muscles and neuromuscular system. Journal of Comparative Neurology, 2020, 528 (1), pp.85-98. 10.1002/cne.24742 . hal-03088556

\section{HAL Id: hal-03088556 https://hal.science/hal-03088556}

Submitted on 30 Nov 2021

HAL is a multi-disciplinary open access archive for the deposit and dissemination of scientific research documents, whether they are published or not. The documents may come from teaching and research institutions in France or abroad, or from public or private research centers.
L'archive ouverte pluridisciplinaire HAL, est destinée au dépôt et à la diffusion de documents scientifiques de niveau recherche, publiés ou non, émanant des établissements d'enseignement et de recherche français ou étrangers, des laboratoires publics ou privés. 


\section{The Drosophila ammonium transporter Rh50 is required for integrity of larval muscles and neuromuscular system}

Short title: Rh50 in the fly neuromuscular system

Mathilde Lecompte ${ }^{1}$, Daniel Cattaert ${ }^{2}$, Alain Vincent ${ }^{3}$, Serge Birman ${ }^{1, *}$ and Baya ChérifZahar $^{1, *}$

${ }^{1}$ Genes Circuits Rhythmes et Neuropathologies, Plasticité du Cerveau, ESPCI Paris, CNRS, PSL University, 75005 Paris, France

${ }^{2}$ Institut des Neurosciences Cognitives et Intégratives d'Aquitaine, CNRS, Bordeaux University, 33076 Bordeaux, France

${ }^{3}$ Centre de Biologie du Développement, Centre de Biologie Intégrative, CNRS, Toulouse University, UPS, Toulouse, France

*Corresponding authors

\section{Acknowledgements}

We would like to thank Drs. Giorgio Matassi, Michèle Crozatier, Henri-Marc Bourbon, and Muriel Boube for their help in the start-up of this project. We thank Dr. Erika Geisbrecht for

This article has been accepted for publication and undergone full peer review but has not been through the copyediting, typesetting, pagination and proofreading process, which may lead to differences between this version and the Version of Record. Please cite this article as doi: 10.1002/cne. 24742 (c) 2019 Wiley Periodicals, Inc.

Received: Dec 07, 2019; Revised: May 30, 2019; Accepted: Jun 21, 2019

This article is protected by copyright. All rights reserved. 
providing the thin RNAi line, Drs. Stephan J. Sigrist and Yaël Grosjean for the gift of GluRIID and GluRIIB antibodies, respectively, and Sandrine Bataille for her help as a student intern in some of the experiments. This work was supported by funding from FRC-Rotary Club, ESPCI Paris and Labex MemoLife (ANR-10-LABX-54 MEMOLIFE) to SB and an AFM Trampoline Grant to SB and BCZ. ML was recipient of a three-year PhD fellowship from PSL Research University and for one supplementary year from Labex MemoLife.

Data Availability Statement: The data that support the findings of this study are available from the corresponding authors upon reasonable request. 


\begin{abstract}
Rhesus glycoproteins ( $\mathrm{Rh50)}$ have been shown to be ammonia transporters in many species from bacteria to human. They are involved in various physiological processes including acid excretion and $\mathrm{pH}$ regulation. Rh50 proteins can also provide a structural link between the cytoskeleton and plasma membranes that maintains cellular integrity. Although ammonia plays essential roles in the nervous system, in particular at glutamatergic synapses, a potential role for Rh50 proteins at synapses has not yet been investigated. To better understand the function of these proteins in vivo, we studied the unique $R h 50$ gene of Drosophila melanogaster which encodes two isoforms, Rh50A and Rh50BC. We found that Drosophila Rh50A is expressed in larval muscles and enriched in the postsynaptic regions of the glutamatergic neuromuscular junctions (NMJs). Rh50 inactivation by RNA interference (RNAi) selectively in muscle cells caused muscular atrophy in larval stages and pupal lethality. Interestingly, Rh50-deficiency in muscles specifically increased glutamate receptor subunit IIA (GluRIIA) level and the frequency of spontaneous excitatory postsynaptic potentials (EPSPs). Our work therefore highlights a new role for Rh50 proteins in the maintenance of Drosophila muscle architecture and synaptic physiology which could be conserved in other species.
\end{abstract}

\title{
Key words
}

Ammonium transporter; Drosophila melanogaster; GluRIIA; larval muscle; neuromuscular junction; muscular atrophy; Rh50 glycoprotein, RRID:BDSC_3605, RRID:BDSC_24650, 
RRID:BDSC_1767, RRID:BDSC_55850, RRID:BDSC_458, RRID:BDSC_7415, RRID:BDSC_32556, RRID:BDSC_27390, Cat\# 55133, RRID:BDSC_55133, RRID :

AB_528269, RRID :AB_2568753, RRID :AB_2569238, RRID : AB_528203, RRID :

AB_2314866, RRID: AB_2534088, RRID: AB_2535849, RRID:AB_2314647,

RRID:SCR_002285 


\section{Introduction}

Ammonia $\left(\mathrm{NH}_{3} / \mathrm{NH}_{4}^{+}\right)$is an indispensable component of all living cells. It is needed for macromolecule biosynthesis and involved in essential physiological processes, such as $\mathrm{pH}$ homeostasis and neurotransmitter production (Bak, 2006; Planelles, 2007; Weiner and Verlander, 2017). In the brain, ammonia is consumed in astrocytes to synthesize glutamine and is produced by neurons to provide glutamate for neurotransmission (Benjamin and Quastel, 1972; Norenberg and Martinez-Hernandez, 1979). In every organism, ammonia concentration is strictly controlled by the regulation of its excretion and neutralization. Given that high concentrations of ammonia are toxic, a disruption of ammonia homeostasis has a serious impact on cells and tissues and is known to lead to neuronal dysfunction and death in humans (Bosoi and Rose, 2009).

Ammonia transporters are membrane proteins that exist in all kingdoms of life. They are required for the absorption of ammonia by microorganisms and plants that extract it directly from environmental nutrients (von Wirén and Merrick, 2004), and for ammonia excretion, which is needed for the regulation of its homeostasis (Wright and Wood, 2009; Biver et al., 2008). Ammonia transporters include Rhesus glycoproteins (Rh50), ammonium/methylammonium proteins (Amt) and methylammonium/ammonium permeases (Mep) (Ludewig et al., 2001), which are differently distributed among prokaryotes and eukaryotes (Matassi, 2017). Amt/Mep proteins are not found in vertebrates whereas Rh50 are present in vertebrates but absent from yeast and plants and rare in prokaryotes. All have been shown to facilitate ammonia uptake and/or excretion (Marini et al., 1997, 2000; Javelle et al., 2004; Wright and Wood, 2009). 
In addition to facilitating ammonia transport, Amt/Mep have been shown to act as ammonia sensor in Saccharomyces cervisiae and Escherichia coli (Lorenz and Heitman, 1998; Javelle and Merrick, 2005), to be involved in signaling pathways in Dictyostelium discoideum (Kirsten et al., 2005) and to have a morphogenic role in Ciona intestinalis (Marino et al., 2007).

$\mathrm{Rh} 50$ have been demonstrated to be $\mathrm{NH}_{3}$ channels in bacteria (Chérif-Zahar et al., 2007; Lupo et al., 2007), fish (Wright and Wood, 2009) and humans (Bakouh et al., 2004; Ripoche et al., 2004; Benjelloun et al., 2005). While Rh50 have the capacity to transport ammonia into the cell, they are rather involved, in vivo, in ammonia excretion (Marini et al., 2000; Wright and Wood, 2009; Weiner and Verlander, 2017). Ammonia excretion that occurs in mammalian kidneys is involved in pH regulation (Lee et al., 2014; Weiner and Verlander, 2017), while in fish gills it participates to osmoregulation (Wright and Wood, 2009).

Besides their transporter function, Rh50 glycoproteins are involved in the maintenance of cell shape. In human red blood cells, Rh50/RhAG associates to other proteins (Rh30, ICAM-4, CD47, glycophorin B) to form a membrane complex (Cambot et al., 2013). This complex is linked by the Rh50 cytoplasmic C-terminus to the spectrin-based cytoskeleton via ankyrin (Nicolas et al., 2003). Mutations in $R H 50 / R H A G$ gene in $\mathrm{Rh}_{\text {null }}$ patients lead to abnormal erythrocyte shape, abnormal cation fluxes and disruption of the normal asymmetric distribution of phospholipids (Nash and Shojania, 1987; Chérif-Zahar et al., 1996; Huang and Ye, 2010), reflecting the role of Rh50/RhAG in the maintenance of cellular architecture.

In invertebrates, both $R h 50$ and Amt genes co-exist, but the relative functions of these two receptors remain to be established. In Aedes albopictus (tiger mosquito), AalRh50 plays a role in 
detoxifying excess ammonia (Wu et al., 2010), in Aedes aegypti (yellow fever mosquito), AeAmt and $A e$ Rh50 proteins are implicated in ammonia excretion within the larval anal papillae (Durant et al., 2017; Durant and Donini, 2018), in Anopheles gambiae (malaria mosquito), it was suggested that AgAmt and $A g R h 50$ are important for ammonia sensitivity in antennae (Pitts et al., 2014) and in Manduca sexta (tobacco hornworm), expression of RhMS has been shown to be higher in tissues involved in ammonia excretion (Weihrauch, 2006). In the fruit fly Drosophila melanogaster, Amt has been shown to be present in auxiliary cells of the olfactory receptor neurons where this transporter is involved in olfactory response to ammonia (Menuz et al., 2014). Here, we addressed the role of the unique Rh50 gene present in Drosophila. We first established that Drosophila Rh50 protein is expressed in larval body wall muscles and enriched at their neuromuscular junction (NMJ). Drosophila NMJs are glutamatergic (Jan and Jan, 1976) and widely used as a model of mammalian excitatory synapses. We therefore addressed the question of the physiological role of Rh50 in the larval neuromuscular system and obtained evidence that both muscle structure and NMJ integrity, and finally fly survival, depend on a normal expression of this ammonia transporter.

\section{Materials and methods}

\subsection{Drosophila strains and culture}

The following Drosophila strains were obtained from the Bloomington Drosophila stock center (BDSC) : $w^{1118}$ (Oregon R) (RRID:BDSC_3605) used as a wild-type control, the RNAi enhancer 
UAS-Dcr-2 (RRID:BDSC_24650), and the driver lines 24B-Gal4 (RRID:BDSC_1767), da-Gal4 (RRID:BDSC_55850), elav-Gal4 (RRID:BDSC_458), repo-Gal4 (RRID:BDSC_7415), c57Gal4 (RRID:BDSC_32556), Mef2-Gal4 (RRID:BDSC_27390), and Mhc-Gal4 (RRID:BDSC_55133). A recombined UAS-mCD8::GFP, UAS-nSyb::GFP strain (named UASmsGFP) (Riemensperger et al., 2013) was used to visualize cellular structure. The RNA interference (RNAi) line targeting Rh50 (UAS-iRh) was obtained from the Vienna Drosophila RNAi Center (ID 9179) and recombined with UAS-Dcr-2 to enhance RNAi activity. To simplify the nomenclature, the combination of UAS-iRh and UAS-Dcr-2 was named UAS-Rh50 ${ }^{\mathrm{RNAi}}$ in the text and figures. Unless specifically described, flies were reared on standard agar-cornmeal-yeast Drosophila medium supplemented with $0.33 \%$ methylparaben as an anti-fungal agent, in a 12:12h light-dark cycle. Eggs were collected from flies kept on grape juice agar plates (25\% grape juice, $2.5 \%$ sucrose, $2.25 \%$ agar, $0.15 \%$ methylparaben) supplemented with yeast paste for $12 \mathrm{~h}$.

\subsection{DNA constructs and generation of transgenic flies}

DmRh50A cDNA was amplified by RT-PCR from Drosophila melanogaster third instar larva RNAs using primers $\mathrm{p} 1$ and $\mathrm{p} 2$ with added restriction sites (Table1). After digestion with EcoRI and BglII, the Rh50A cDNA was inserted into pUASTattB (Bischof et al., 2007). The human RhCG cDNA was amplified from $p T 7 T S-R h C G$ clones (Bakouh et al., 2004) using primers $\mathrm{p} 3$ and p4 with added restriction sites (Table1). The RhCG cDNA was inserted between the BglII and XhoI sites in pUAST (Brand and Perrimon, 1993). Constructs were sequenced (GATC 
Biotech) and sent to BestGene Inc. for Drosophila germline transformation. UAS-DmRh50A was inserted into the attP40 docking site on chromosome 2, and $U A S-H s R h C G$ by random insertion.

\subsection{Reverse transcription-coupled PCR and qPCR}

Total RNAs were extracted from 10 third-instar larvae using QIAzol Lysis reagent (Qiagen). The first-strand cDNAs were generated from $0.5 \mu$ R RA by Maxima First Strand cDNA Synthesis

Kit (ThermoFischer Scientific). The relative levels of Rh50 mRNA in control and $R h 50^{\mathrm{RNAi}}$ larvae were estimated by semi-quantitative PCR: duplex PCR was performed using the primer pairs p5-p6 and p10-p11 (Table 1) for Rh50 (480 nt) and Rp49 (189 nt), respectively, in $50 \mu l$ of reaction mixture using PrimeStar Max DNA polymerase (Takara). Rp49 encodes a ribosomal protein and was used as internal control. The program (30 cycles) included $10 \mathrm{~s}$ denaturation at $98^{\circ} \mathrm{C}, 5 \mathrm{~s}$ annealing at $55^{\circ} \mathrm{C}$ and $10 \mathrm{~s}$ elongation at $72^{\circ} \mathrm{C}$. PCR product levels were estimated after electrophoresis by densitometry with the Fiji software (Fiji, RRID:SCR_002285) (Schindelin et al., 2012). Quantitative RT-qPCR assays were performed using the LightCycler 480 SYBR Green I Master mix (Roche LifeScience). Reactions were performed in triplicate, from three independent RNA extractions. Rp49 was used as internal control for normalization of mRNA levels. The primers used for the amplification of Rh50A or Rh50BC were p7 (common to all Rh50 transcripts) coupled either with p8 or p9, respectively (Table 1).

\section{$2.4 \quad$ Immunohistochemistry}

This article is protected by copyright. All rights reserved. 
Third-instar larvae were collected, rinsed in water and dissected in hemolymph-like saline solution (HL3) (in mM: $70 \mathrm{NaCl}, 5 \mathrm{KCl}, 1.5 \mathrm{CaCl}_{2}, 20 \mathrm{MgCl}_{2}, 10 \mathrm{NaHCO}_{3}, 120$ sucrose and 5 HEPES at pH 7.6) (Cattaert \& Birman, 2001). Body wall muscles were fixed with $4 \%$ paraformaldehyde (PFA, ThermoFischer Scientific) for 20 min, or, alternatively, with Bouin's fixative solution for GluRIIA antibody for $35 \mathrm{~min}$. The samples were blocked by incubation in phosphate-buffered saline containing 0.5\% Triton X-100 (PBST) and 2\% bovine serum albumin

for $1 \mathrm{~h}$. Larval muscle preparations were exposed to the primary antibodies during $24 \mathrm{~h}$, and then after several washes in PBST, to the secondary antibodies during $12 \mathrm{~h}$ at $4^{\circ} \mathrm{C}$. Samples were mounted in ProLong Gold Antifade reagent (Invitrogen Molecular Probes) and scanned on a Nikon A1R confocal microscope.

\subsection{Antibody characterization}

Antibodies used in this study are listed in Table 2.

Anti-Rh50: The polyclonal anti-Rh50 antibody was produced in rabbit by co-injection of two synthetic peptides: ${ }_{43} E_{\text {EAGSANEHVSKYPQFQD }}$ and ${ }_{423} \mathrm{NFRNLKKDEHHQDEHYWE}_{440}$. The specificity of the antibody was verified in the embryo by comparing in situ hybridization and immunohistochemistry patterns: the antiserum reactivity fully matched the pattern of Rh50 RNA expression. In larval muscles, the Rh50 immunoreactivity is localized at the NMJ and in extrasynaptic regions of the muscles. When Rh50 was overexpressed in muscles, the immunoreactivity increased substantially and matched the expression pattern of the endogenous 
Rh50 protein (Figure 1f). Anti-GluRIIA (Developmental Studies Hybridoma Bank (DSHB) Cat\# 8B4D2 (MH2B), RRID:AB_528269RRID) : mouse monoclonal anti-GluRIIA antibody labels larval neuromuscular junctions. GluRIIA immunoreactivity appears as puncta located opposite to the active zone at synaptic boutons. This antibody detected the same muscular pattern as an antimyc antibody in flies expressing a myc-tagged GluRIIA transgene (Marrus et al., 2004). AntiGluRIID (Qin G; J Neurosci. 2005 Cat\# GluRIID, RRID:AB_2569238) : rabbit polyclonal antiGluRIID antibody, a gift from Dr. Stephan J. Sigrist (Freie Universität Berlin, Germany), labels larval neuromuscular junctions. It recognizes the essential D subunit of the glutamate receptors at the postsynaptic densities (Qin et al., 2005). Anti-GluRIIB (Marrus SB; J Neurosci. 2004 Cat\# GluRIIB, RRID:AB_2568753) : rabbit polyclonal anti-GluRIIB antibody, a gift from Dr. Yaël Grosjean (Centre des Sciences du Goût et de l’Alimentation, Dijon, France), labels larval neuromuscular junctions. It recognizes the GluRIIB receptors at the postsynaptic densities. The staining is not detectable in flies null for DGluRIIB but it appears normal in flies null for DGluRIIA (Marrus et al., 2004). Anti-Dlg (DSHB Cat\# 4F3 anti-discs large, RRID:AB_528203): mouse monoclonal anti-Dlg antibody stains the third-instar body wall muscles. The immunoreactivity is concentrated on the postsynaptic side of the neuromuscular junction labeling a specialized membrane network called the subsynaptic reticulum that surrounds synaptic boutons. Immunoreactivity is also observed in extrasynaptic regions (Parnas et al., 2001). AntiBrp (DSHB Cat\# nc82, RRID:AB_2314866) : mouse monoclonal anti-Bruchpilot (Brp) recognizes brain neuropil and synaptic active zones. This antibody recognizes two protein bands in western blot of homogenized Drosophila heads (170 and $190 \mathrm{kDa}$ ) that correspond to the Brp 
protein. The nc82 signal decreased in situ after RNAi-mediated brp inactivation (Wagh et al., 2006). Anti-GFP: Monoclonal anti-GFP (DSHB 12A6) antibody was used for enhancing the GFP signal of mCD8::GFP and nSyb::GFP. It is a mouse antiserum raised against the recombinant wild-type GFP from Aequorea victoria. In larvae in which GFP is ectopically expressed in neurons, enhancement of the GFP signal with the anti-GFP was consistent with the expected pattern of the driver used. FITC-HRP (Jackson ImmunoResearch Labs Cat\# 123-095021, RRID:AB_2314647) : The FITC-conjugated goat anti-horseradish peroxidase (HRP) antibody was used as a neuronal marker since it specifically binds to neuronal membrane in Drosophila (Jan and Jan, 1982).

\subsection{Quantification of immunofluorescence intensity}

Immunofluorescence intensity in muscles and NMJs was quantified on Z-projections (max intensity) of confocal stacks of muscles 6 and 7, segment A3, using the Fiji software. These confocal stacks were obtained using constant laser power and gain, and same interstack step for all acquisitions and all genotypes. For quantification of muscles, the region of interest (ROI) was the whole muscular fiber, excluding the NMJ. For the NMJs, the ROI was the entire NMJ and ROI was restricted to NMJ staining using the Fiji “threshold” tool. The threshold value was manually set to exclude non-NMJ staining.

\subsection{Quantification of NMJ area, synaptic boutons and muscle size}


To measure area at the NMJ, the Fiji “analyze particles” tool was used to define ROIs corresponding to Brp or Dlg fluorescent immunostaining on Z-projections (sum) of confocal stacks in muscles 6 and 7. The area of each ROI was then summed to obtain the total staining area. The number of synaptic boutons (type Ib and Is) was manually counted on Z-projections (max) of confocal stacks of the NMJ of muscles 6 and 7 stained with anti-Dlg antibody. Type Is can be distinguished from type Ib by a lighter Dlg staining around the boutons. For muscle size measurement, third instar larvae were dissected and the body wall muscles processed for immunofluorescence with anti-Dlg antibody (1:500), then imaged with the confocal microscope. The width of muscles 6 and 7, segment A3, was measured with the Fiji software.

\subsection{Protein extraction and western blotting}

Twenty third instar larvae were dissected in HL3 and their carcasses (body wall muscles and cuticle) were homogenized in $300 \mu$ l RIPA buffer (Sigma-Aldrich) containing protease inhibitor (cOmplete Protease Inhibitor Cocktail, Roche Diagnostics), using bead tubes and a Minilys apparatus (Bertin Technologies). Proteins samples and western blot were performed as described (Issa et al., 2018). Briefly, the extracted proteins were mixed with LDS sample buffer and reducing agent (NuPAGE, Invitrogen), heated at $70^{\circ} \mathrm{C}$ for $5 \mathrm{~min}$, and separated in $4-12 \%$ Novex NuPAGE Bis-Tris precast polyacrylamide gels (Life Technologies) following the manufacturer’s protocol in a MOPS-SDS running buffer. A semi-dry transfer was done onto Polyvinylidene (PVDF) difluoride membranes (Amersham Hybond P $0.45 \mu \mathrm{m}$ ) using a Hoefer TE77 apparatus. The mouse monoclonal anti-GluRIIA (8B4D2) and anti- $\alpha$-tubulin (12G10) from DSHB were 
used at 1:50 and 1:500 dilution, respectively. Immunolabeled bands were revealed by ECL RevelBlOt Intense (Ozyme) as chemiluminescent HRP substrate and digitally acquired using ImageQuant TL software (GE Healthcare Life Science). Densitometry measures were made with the Fiji software and normalized to the $\alpha$-tubulin measures as internal controls.

\subsection{Larval locomotion and survival assays}

For locomotion assay, third instar larvae (100 \pm 5 h after egg laying) were collected and briefly rinsed in water to remove traces of food. After an equilibration period of $30 \mathrm{~s}$ on a 14.5-cm diameter agar plate (2\% wt/vol), larval crawling was examined under a dissection microscope. The agar plate was placed on a graphic paper with a $2 \mathrm{~mm}^{2}$ grid, then the number of peristaltic waves (full anterior to posterior movement $=1$ wave) and the distance crawled (the number of grid lines crossed in $5 \mathrm{~min}$ ) were scored. The locomotion stride (mm/peristaltic wave) was obtained by dividing the distance covered in 5 min by the number of peristaltic waves made during this period.

To monitor larval survival, adults were allowed to lay eggs on agar and grape juice plates. $24 \mathrm{~h}$ after egg-laying, 20 first-instar larvae were placed in regular fly food vials. Five days later, the number of pupae was reported. Larval survival was scored in each vial as the number of pupae formed divided by the number of larvae introduced in the vial and was named rate of pupa formation. In each experiment, a minimum of 8 vials per genotype was used to assess the survival score. 


\subsection{Electrophysiology}

Electrophysiological recordings were generally performed as previously described (Cattaert and Birman, 2001). Third instar larvae were collected from the fly medium and rinsed in water, then pinned into a silicon-coated Petri dish and dissected in HL3 solution. The cuticle was opened dorsally, the gut, fat body and central nervous system removed, and body wall muscles were left intact. Thin glass electrodes, of mean resistance $23 \mathrm{M} \Omega(7-36 \mathrm{M} \Omega$ ) filled with $3 \mathrm{M} \mathrm{KCl}$ were used for intracellular recording of muscle 6 (ventral longitudinal muscle) in abdominal segments A3, A4 and A5. Excitatory postsynaptic potentials (EPSPs) were recorded in current clamp (CC) and excitatory postsynaptic currents (EPSCs) in voltage clamp (VC, -70 mV) using an Axoclamp 2B (Axon Instruments, Inc., Foster City, CA) and observed with a digital oscilloscope (Yokogawa DL 1200, Tokyo, Japan). Signals were converted by an A/D interface (Cambridge Electronic Device, CED 1401Plus, Cambridge, UK) at a sampling rate of $16 \mathrm{kHz}$ in CC and at 60 $\mathrm{kHz}$ in VC. The SPIKE2 (CED) software was used for recording analysis.

\subsection{Statistical analysis}

Statistical analysis was performed with GraphPad Prism 6 software. When two genotypes were compared to each other, the Student's t test was used. When three genotypes or more were compared, ANOVA was used with Tukey (one-way ANOVA) or Sidak (two-way ANOVA) posthoc tests for pairwise comparisons. If one of Bartlett's and Brown-Forsythe's test indicated variance inequality, then Kruskall-Wallis (non-parametric) followed by Dunn's post-hoc test 
were used instead of ANOVA. Mean values and standard error of the mean (SEM) are reported in the text and figures.

\section{$3 \quad$ Results}

\subsection{Alternative splicing of Drosophila $R h 50$ encodes two protein isoforms}

Analysis of the Drosophila melanogaster genome (The FlyBase Consortium, 2003) revealed the presence of a single $R h 50$ gene (DmRh50 or $R h 50$ ). Alternative splicing produces three $R h 50$ transcripts: Rh50A, Rh50B and Rh50C (Figure 1a). Rh50B and Rh50C only differ by the size of their 3'-UTR, most likely from the use of different polyadenylation sites. In both $R h 50 B$ and Rh50C, the last nucleotide of the Rh50A stop codon and the following 237 nucleotides are eliminated (Figure 1a). As a consequence, $R h 50 B$ and $R h 50 C$ mRNAs have 11 additional codons compared to Rh50A, and they encode the same protein designated below as Rh50BC. Rh50A (449 amino acids) and Rh50BC (460 amino acids) are predicted to form 11 transmembrane domains with an extracellular N-terminus and an intracellular C-terminus. Drosophila Rh50 is expressed during all stages of development (data not shown) and the three mRNAs are detected in third-instar larvae, the predominant isoform being $R h 50 \mathrm{~A}$ (Figure $1 \mathrm{~b}$ ).

\subsection{Rh50A is expressed in larval muscles and enriched at the NMJ}

To determine more precisely where Rh50 is expressed, a polyclonal antibody was raised against two hydrophilic peptides of 18 amino acids, from the $\mathrm{N}$ - and C-terminal regions of the Rh50 
protein, respectively. Immunofluorescence labeling showed that the Rh50 protein is expressed in larval body wall muscles and enriched at the NMJs (Figure 1c). NMJs were identified by immunostaining of muscle with an anti-HRP antibody, a specific neuronal marker in Drosophila (Jan and Jan, 1982). We then examined the precise localization of Rh50 at the larval NMJ by double staining with presynaptic and postsynaptic markers. Expression of membrane-bound mCD8/nSyb-GFP (msGFP) (Riemensperger et al., 2013) under control of elav-Gal4, a panneuronal driver, was used to label specifically the motor neurons and their synaptic endings. As shown in Figure 1d, the Rh50 immunoreactivity surrounded the synaptic boutons, suggesting a postsynaptic localization. To verify this observation, we stained muscle preparations for Discs large (Dlg), a scaffolding protein expressed in the muscle subsynaptic reticulum (SSR) and a reliable postsynaptic marker (Lahey et al., 1994). Co-immunostaining of Rh50 and Dlg indicated that Rh50 co-localized with Dlg at the periphery of the synaptic boutons (Figure 1e).

Overexpression of Rh50 in muscles using the $24 B$-Gal4 driver (24B $>R h 50$ flies) increased Rh50 immunostaining intensity in the postsynaptic part of the NMJ (Figure 2f). These data indicate that Rh50 is a postsynaptic protein at the larval NMJ.

\subsection{Rh50 inactivation leads to developmental defects and pupal lethality}

No Rh50 mutant is currently available in Drosophila. To investigate possible roles of DmRh50 in specific larval tissues, we used RNAi to silence its expression. In a first set of experiments, we ubiquitously co-expressed a Rh50 interfering RNA (iRh) (Figure 1a) and the RNAi enhancer Dicer-2 (Dcr-2) in larval cells using the ubiquitous $d a-G a l 4$ driver ( $d a>i R h, D c r-2$, referred to 
here as $d a>R h 50^{\mathrm{RNAi}}$ ). $d a>R h 50^{\mathrm{RNAi}}$ led to a nearly total loss (97\% decrease) of $R h 50$ mRNA (Supporting Information Figure S1a-c) and resulted in lethality that occurred at different stages of development according to the temperature at which the flies were reared. At $29^{\circ} \mathrm{C}$, when Gal4 is at its maximal activity, larvae died before they reached the third-instar stage. At $25^{\circ} \mathrm{C}$, the larvae survived until the pupal stage. We observed that Rh50-deficient pupae were longer and thinner than wild-type pupae (Figure 2a). A similar phenotype was previously described in Drosophila and explained by muscular dysfunction and the inability for larvae to use muscle contraction to shorten the cuticle prior to pupariation (Ball et al., 1985; LaBeau-DiMenna et al., 2012). This observation, together with Rh50 localization at the NMJ suggested that Rh50 function is required for normal muscle physiology.

To address this question further, we used tissue-specific RNAi to reduce Rh50 level either in neural cells or in muscle. We tested several neuronal or glial drivers and four muscular drivers (24B-Gal4, Mef2-Gal4, C57-Gal4, and Mhc-Gal4). Only the muscular drivers replicated the phenotypes obtained with $d a-G a l 4$, suggesting that the puparial phenotype indeed derived from muscle dysfunction. We thereafter consistently used 24B-Gal4 to further characterize Rh50 inactivation in body wall muscles (Supporting Information Figure S1b,d,e). Rh50 protein level at the NMJ was found by immunostaining to be significantly reduced ( 3-fold) in $24 B>R h 50^{\mathrm{RNAi}}$ larvae relative to the controls (Supporting Information Figure S1d,e). Although Rh50 expression appears mainly postsynaptic, an expression of the protein in presynaptic and/or glial cells could not be excluded. The remaining Rh50 immunoreactivity detected in $24 B>R h 50^{\mathrm{RNAi}}$ muscle preparations could then in part correspond to extramuscular expression. We found that muscles of 
dissected Rh50-deficient third-instar larvae were narrower and more elongated than wild-type muscles and displayed an irregular shape that resembled a dystrophy (Figure 2b). We further characterized this phenotype by measuring muscle width of two external ventral longitudinal muscles, muscle 6 and 7 (Figure 2b,c). Rh50-deficient muscles were significantly narrower compared to controls $\left(24 B>R h 50^{\mathrm{RNAi}}: 134.5 \pm 2.8 \mu \mathrm{m}, 24 \mathrm{~B}-\mathrm{Gal} 4 /+: 169.9 \pm 5 \mu \mathrm{m}, p<0.01\right)$ (Figure 2c). These phenotypes could not be rescued by overexpression of DmRh50 (data not shown), whereas expression of the human orthologue $R h C G$, which is not a target of $R h 50^{\mathrm{RNAi}}$, induced a significant increase in muscle width $\left(24 B>R h 50^{\mathrm{RNAi}}, R h C G: 152.4 \pm 2.4 \mu \mathrm{m} ; p<0.05\right)$, indicating that muscle atrophy had been partially rescued (Figure 2c). Furthermore, the rate of

pupa formation increased in $24 B>R h 50^{\mathrm{RNAi}}$; $R h C G$ flies compared to $24 B>R h 50^{\mathrm{RNAi}}$, indicating that a partial rescue of larval survival also occurred in the presence of RhCG (Figure 2d). These results suggest that the muscle and lethality phenotypes are a direct consequence of $R h 50$ inactivation in muscles.

\subsection{Both reduced Rh50 level and high ammonia concentrations impact GluRIIA expression}

To further characterize the morphological changes at the NMJ when Rh50 level is reduced, we examined pre- and post-synaptic markers and the number and size of synaptic boutons. In $24 B>R h 50^{\mathrm{RNAi}}$ flies, the area of Dlg immunostaining at the larval NMJ appeared similar compared to the wild type (Figure 3a). We also observed that a deficiency in Rh50 expression in muscles had no effect on the size of active zone areas (stained with an anti-Brp antibody) (Figure 
3b). Moreover, Rh50 deficiency did not change the number of synaptic boutons (type Ib and Is) (Figure 3c,d). This shows that the general morphology of the NMJ and particularly of its presynaptic components are not affected by Rh50 deficiency.

The localization of the Rh50 membrane protein at the periphery of synaptic boutons raised the possibility of its association with other postsynaptic NMJ proteins. Prominent postsynaptic markers are the GluRII ionotropic glutamate receptors that play a central role in excitatory synaptic transmission. These receptors are heterotetramers containing either the GluRIIA or GluRIIB subunit plus three constitutive subunits: GluRIID, GluRIIC and GluRIIE (Marrus et al., 2004). Interestingly, we found that silencing $R h 50$ expression in muscles triggered a significant increase in GluRIIA levels in the whole muscle (Figure 3e). This was demonstrated by quantifying GluRIIA on western blots of larval carcasses which mainly contains body wall muscles and cuticles; GluRIIA level was found to be more than 8-fold increased compared to the controls (Figure 3f,h). We also measured the intensity of GluRIIA immunostaining at the NMJs and on extrasynaptic regions of the muscle. As shown in Figure 3g, GluRIIA signal increased in muscles of $24 B>R h 50^{\mathrm{RNAi}}$ larvae but not at the NMJ compared to the controls. In contrast, we did not observe a change in GluRIIB and GluRIID abundance, either at NMJs or extrajunctional regions, in $24 B>R h 50^{\mathrm{RNAi}}$ larvae (Supporting Information Figure S2a-f).

To examine if the increase of GluRIIA may be induced by the muscular atrophy, we looked at the GluRIIA expression in thin-deficient larvae (mutants for an ortologue of mammalian Tripartite motif-containing protein 32 (TRIM32)) that exhibit muscular atrophy and evolve toward a similar pupal shape phenotype than Rh50-deficient larvae (LaBeau-DiMenna et al., 2012). We observed 
in all control larvae $\left(24 \mathrm{~B} /+, R h 50^{\mathrm{RNAi} /+}\right.$, thin $\left.^{\mathrm{RNAi} /+}\right)$ an accumulation of GluRIIA immunoreactivity (as shown for 24B/+ in Figure 4) at attachment sites of lateral muscles (arrows) and at muscle-muscle junctions (asterisks). These specific sites of GluRIIA expression were not visible in $24 B>R h 50^{\mathrm{RNAi}}$ larvae (Figure $4 \mathrm{~b}$ ). GluRIIA localization in muscles appeared uniform and widespread, or in the form of plaques (Figure 3e, 4b). In $24 B>$ thin $^{\mathrm{RNAi}}$ larvae, the GluRIIA localization at attachments sites were in contrast still present but less structured (Figure 4c). Traces of dispersed GluRIIA immunofluorescence could be observed in a few $24 B>$ thin $^{\mathrm{RNAi}}$ but never a high level of expression as it could be seen in Rh50-deficient larvae. These results indicate that disruption of the muscular structure disturbs the localization of extrajunctional GluRIIA in muscles but is likely not the only cause for its higher expression in Rh50-deficient larvae.

Since Rh50 is presumably an ammonium transporter, it could be involved in the regulation of ammonia homeostasis in muscles. We therefore examined whether modification of ammonia concentration can also affect GluRIIA expression in larval muscles. Wild-type larvae were exposed to high ammonia concentration $(350 \mathrm{mM})$ in the food, from the first to the third stage. This concentration is similar to that used to study ammonia tolerance in Drosophila (Borash et al., 2000; Belloni et al., 2018). The treated larvae consistently showed a ca. $26 \%$ and $14 \%$ decrease in synaptic and extrasynaptic GluRIIA levels in muscles, respectively (Supporting Information Figure S3a,b). Interestingly, we also observed a 3-fold increase in Rh50 mRNA in whole larvae fed with ammonia compared to the non-treated larvae (Supporting Information Figure S3c). This suggests that hyperammonemia regulates negatively GluRIIA expression and 
that removing Rh50 lowers ammonia transport across the sarcolemmal membrane, leading to higher GluRIIA expression. Thus, both Rh50 expression and ammonia concentration regulation are required for the maintenance and/or localization of GluRIIA in the body wall muscle, suggesting that Rh50 contributes to ammonia transport and homeostasis in the muscles of Drosophila larvae.

\subsection{Loss of Rh50 induces defects in larval locomotion}

The muscle atrophy and increase in GluRIIA level observed in $R h 50^{\mathrm{RNAi}}$ flies is expected to alter larval locomotion. We noticed that the crawling of the $24 \mathrm{~B}>R h 50^{\mathrm{RNAi}}$ larvae was indeed impaired since they apparently needed more peristaltic waves than wild-type larvae to cross the same distance. A locomotor assay was designed to calculate the locomotor stride, which corresponds to the distance traveled during one peristaltic wave (Figure 5a). Rh50-deficient larvae displayed a ca. $40 \%$ decrease in stride magnitude compared to the controls (Figure 5b). This defect was also observed when $R h 50$ was inactivated by RNAi using other muscular drivers, such as $c 57-G a l 4$ and Mhc-Gal4 (not shown). In contrast, this defect was not observed when Rh50 inactivation was performed using neuronal or glial drivers (Figure 5c), suggesting that Rh50 function is specifically required in muscles and/or postsynaptic region of the NMJ to ensure a normal crawling.

\subsection{Electrophysiological activity of the NMJ is altered in the absence of Rh50}


In order to investigate putative neurotransmission defects induced by Rh50 inactivation, we performed electrophysiological recording of spontaneous NMJ activity, by intracellular

recordings in muscle 6. The mean amplitude of miniature EPSCs (mEPSCs) in $24 B>R h 50^{\mathrm{RNAi}}$ larvae was lower but not significantly different than those of control larvae $(-0.669 \pm 0.064 \mathrm{nA} v \mathrm{~s}$ $-0.810 \pm 0.084 \mathrm{nA}$; Student's t test: $p>0.05$ ) (Figure 6a-c). In contrast, we found that the mean frequency of miniature EPSPs (mEPSPs) was markedly (ca. 53\%) and significantly increased in $R h 50^{\mathrm{RNAi}}$ larvae compared to controls $(4.665 \mathrm{~Hz} \pm 0.500$ vs $3.054 \mathrm{~Hz} \pm 0.360$ Student’s t test: $p=$ 0.020) (Figure 6a,b,d).

\section{Discussion}

Rh50 proteins were shown to be ammonia transporters in many species from bacteria (ChérifZahar et al., 2007) to humans (Marini et al., 2000), indicating that this function was conserved during evolution. They are involved in essential physiological processes such as ammonia excretion (Biver et al., 2008; Wright and Wood, 2009; Durant et al., 2017), and the regulation of acid-base homeostasis (Weiner and Verlander, 2017). Human Rh50 glycoproteins are also implicated in cell structure maintenance via their interaction with ankyrins and the cytoskeleton (Nicolas et al., 2003; Lopez et al., 2005). Here we show that the only Drosophila Rh50 gene is expressed in muscles and enriched at the NMJ in larval stages. Interestingly, Drosophila NMJs are glutamatergic synapses that share similar developmental and functional mechanisms with 
those of the vertebrate central nervous system (Menon et al., 2013). The aim of our study was therefore to examine whether Rh50 is implicated in neuromuscular physiology.

\subsection{Rh50 is required for the development and maintenance of muscle structure}

We detected Rh50 expression in the larval muscles and in the postsynaptic part of the NMJ. Rh50 is present at the periphery of synaptic boutons where it partially colocalizes with the scaffolding protein Dlg. Inactivation of $R h 50$ by RNAi targeted to muscles led to muscular atrophy in larval stages, and pupal lethality. Such a phenotype suggests that Rh50 could be involved in the development and maintenance of muscle cell structure. This phenotype of cellular structural instability is reminiscent of morphological abnormalities observed in Drosophila models for muscular dystrophies (Kreipke et al., 2017) and of structural defects in $\mathrm{Rh}_{\text {null }}$ red blood cells in human, in which Rh50 glycoprotein is missing (Nash and Shojania, 1987; Chérif-Zahar et al., 1996). In mammal cells, Rh50 has been shown to interact with the cytoskeleton (Nicolas et al., 2003; Lopez et al., 2005). The structural abnormalities induced by the lack of Rh50 in fly muscles could therefore be explained by a disruption of an important link that stabilizes cellular structure. In Rh50-deficient larvae, this structural defect could be partially rescued by ectopic expression in muscles of the human homolog RhCG. It is likely that Drosophila Rh50 interacts with structural proteins to maintain the architecture of muscle cells.

Muscular atrophy could also be a consequence of an imbalance in ammonia homeostasis. A change in metabolic composition is critical to many physiological processes and can compromise signaling pathways. Loss of muscle mass might result from an increase in protein degradation 
involving the autophagy-lysosome pathway (Zhao et al., 2007). Indeed, hyperammonemia has been shown to increase autophagy and can damage muscle structure (Qiu et al., 2012). A combination of the two events, structural defect and ammonia imbalance, in the Rh50-deficient flies, could severely compromise muscle function and lead to myodegeneration.

\subsection{Rh50 function is required for normal muscle physiology}

Here we show that larval locomotion is impaired in Rh50 knockdown larvae as they require more peristaltic waves than wild type to crawl along the same distance. This suggests that muscle contractions are less efficient, consistent with the muscle structural abnormalities. We also observed that muscle atrophy and pupal lethality were always correlated. Mutations affecting structural muscle proteins that are associated to a muscular atrophy and pupal lethality were previously reported in Drosophila (LaBeau-DiMenna et al., 2012; Clark et al., 2007). In larvae, muscle contractions are required to perform pupal ecdysis, a process that follows pupation and that is required for head eversion (Bainbridge and Bownes, 1981). In mlp84B mutants (a muscle LIM protein), this process does not take place, which is reflected by the fact that an air pocket remains at the most posterior part of the pupa whereas it is translocated at the most anterior part in wild-type pupae (Clark et al., 2007). Rh50-deficient pupae also feature this phenotype, suggesting that muscular impairment is the cause of pupal lethality.

\subsection{Consequence of Rh50 deficiency on GluRIIA expression}


Here we show that Rh50-deficient larvae exhibited overexpression of the glutamate receptor subunit GluRIIA in larval muscles, but not of the constitutive GluRIID subunit. Moreover the increase in GluRIIA is not associated to a decrease in GluRIIB subunit, indicating that this increase does not correspond to a change in the nature of the glutamate receptors. Temporal restriction of Rh50 inactivation indicated that this increase may correlate with muscle atrophy (data not shown). Muscular atrophy could promote GluRIIA overexpression as an attempt to compensate for a reduced excitation-contraction coupling. Here, we show that the attachment sites of muscles to the cuticle-associated tendon express high level of GluRIIA. These "GluRIIA pockets” are lacking in Rh50 knockdown larvae possibly due to a disruption of muscular architecture. The loss of stable structures containing GluRIIA could induce a misregulation of this subunit and its overexpression in muscles of $R h 50^{\mathrm{RNAi}}$ flies. This may also occur, however to a lesser extend, in the thin-deficient larvae that also exhibit muscular atrophy. Overexpression of glutamate receptor subunits has never been reported to our knowledge in fly mutants showing muscle atrophy.

\subsection{Role of ammonia buffering in GluRIIA regulation}

Our results indicate that a structural defect may have an impact on GluRIIA expression in muscle, but it is probably not the only cause of its higher expression in Rh50-deficient larvae. To investigate if a disturbance of intramuscular ammonia level may affect GluRIIA expression, we exposed wild-type larvae to hyperammonemia and determined GluRIIA levels in muscle and NMJ. GluRIIA protein levels were significantly decreased when larvae were raised in high 
concentrations of $\mathrm{NH}_{4} \mathrm{Cl}$. Overall, our results show that high level of ammonia downregulates GluRIIA expression, whereas Rh50 deficiency, on the opposite, increases it. The lack of Rh50 could decrease ammonia transport leading to higher GluRIIA expression, although more work is needed to test this hypothesis. Nevertheless, these observations, together with the fact that Rh50 is strongly upregulated in larvae exposed to ammonia, provide significant evidence that Rh50 plays a central role in ammonia buffering in Drosophila.

\subsection{Effects of Rh50 knockdown on NMJ physiology}

The increase in miniature current frequency induced by $R h 50$ inactivation could be due to a change in NMJ morphology (Stewart et al., 1996). However, staining for the active zone marker Brp and the postsynaptic marker Dlg did not reveal any difference in NMJ morphology and synaptic bouton number. Therefore, the increase in frequency was more likely caused by a higher probability of vesicle release from the motor neuron. It was previously reported that vesicle release probability depends on presynaptic $\mathrm{pH}$, and that a higher mEPSC frequency reflects its decrease (Caldwell et al., 2013). Our results therefore suggest a potential involvement of Rh50 in synaptic $\mathrm{pH}$ homeostasis at the NMJs.

In conclusion, our study shows that the Drosophila Rh50 gene is necessary for muscle development and normal functioning of the neuromuscular system. Whether disruption of ammonia homeostasis and/or defects in membrane-cytoskeleton interactions are responsible for muscular atrophy in Rh50-deficient larvae remains to be investigated. Glutamate receptor 
upregulation and increase in spontaneous mEPSP frequency suggest that Rh50 act as an ammonia transporter in the Drosophila larval neuromuscular system. Rh50 could therefore be involved in the regulation of ammonia level and $\mathrm{pH}$ in muscles and synapses, a function that could also be conserved in vertebrates. Several major signaling pathways, such as Wnt/ $\beta$-catenin signaling, are known to be modulated by intracellular pH (Strubberg et al., 2017). Future studies could therefore examine whether ammonia imbalance can affect muscular development through these pathways as well.

\section{References}

Augustin, H., Grosjean, Y., Chen, K., Sheng, Q., \& Featherstone, D. E. (2007). Nonvesicular release of glutamate by glial xCT transporters suppresses glutamate receptor clustering in vivo. The Journal of Neuroscience, 27(1), 111-123. doi:10.1523/JNEUROSCI.4770$\underline{06.2007}$

Bainbridge, S. P., \& Bownes, M. (1981). Staging the metamorphosis of Drosophila melanogaster. Development, 66(1), 57-80.

Bak, L. K., Schousboe, A., \& Waagepetersen, H. S. (2006). The glutamate/GABA-glutamine cycle: aspects of transport, neurotransmitter homeostasis and ammonia transfer. Journal of Neurochemistry, 98(3), 641-653. doi:10.1111/j.1471-4159.2006.03913.x 
Bakouh, N., Benjelloun, F., Hulin, P., Brouillard, F., Edelman, A., Chérif-Zahar, B., \& Planelles, G. (2004). $\mathrm{NH}_{3}$ is involved in the $\mathrm{NH}_{4}{ }^{+}$transport induced by the functional expression of the human Rh C glycoprotein. The Journal of Biological Chemistry, 279(16), 15975-15983. doi: $\underline{10.1074 / j b c . M 308528200}$

Ball, E., Ball, S. P., \& Sparrow, J. C. (1985). A mutation affecting larval muscle development in Drosophila melanogaster. Developmental Genetics, 6(2), 77-92.

Belloni, V., Galeazzi, A., Bernini, G., Mandrioli, M., Versace, E., \& Haase, A. (2018). Evolutionary compromises to metabolic toxins: Ammonia and urea tolerance in Drosophila suzukii and Drosophila melanogaster. Physiology \& Behavior, 191, 146-154. doi: 10.1016/j.physbeh.2018.04.021

Benjamin, A. M., \& Quastel, J. H. (1972). Locations of amino acids in brain slices from the rat. Tetrodotoxin-sensitive release of amino acids. The Biochemical Journal, 128(3), 631-646. doi: $\underline{10.1042 / b j 1280631}$

Benjelloun, F., Bakouh, N., Fritsch, J., Hulin, P., Lipecka, J., Edelman, A., ... Chérif-Zahar, B. (2005). Expression of the human erythroid Rh glycoprotein (RhAG) enhances both $\mathrm{NH}_{3}$ and $\mathrm{NH}_{4}{ }^{+}$transport in HeLa cells. Pflugers Archiv: European Journal of Physiology, 450, 155-167. doi: 10.1007/s00424-005-1381-y

Bischof, J., Maeda, R. K., Hediger, M., Karch, F., \& Basler, K. (2007). An optimized transgenesis system for Drosophila using germ-line-specific phiC31 integrases. Proceedings of the National Academy of Sciences of the United States of America, 104(9), 3312-3317. doi:10.1073/pnas.0611511104 
Biver, S., Belge, H., Bourgeois, S., Van Vooren, P., Nowik, M., Scohy, S., ... Marini, A. M. (2008). A role for Rhesus factor Rhcg in renal ammonium excretion and male fertility. Nature, 456(7220), 339-343. doi:10.1038/nature07518

Borash, D., Pierce, V., Gibbs, A., \& Mueller, L. (2000). Evolution of ammonia and urea tolerance in Drosophila melanogaster: resistance and cross-tolerance. Journal of Insect Physiology, 46(5), 763-769.

Bosoi, C. R., \& Rose, C. F. (2009). Identifying the direct effects of ammonia on the brain. Metabolic Brain Disease, 24(1), 95-102. doi:10.1007/s11011-008-9112-7

Brand, A. H., \& Perrimon, N. (1993). Targeted gene expression as a means of altering cell fates and generating dominant phenotypes. Development, 118(2), 401-415.

Cambot, M., Mazurier, C., Canoui-Poitrine, F., Hebert, N., Picot, J., Clay, D., ... Cartron, J.P. (2013). In vitro generated $\mathrm{Rh}$ (null) red cells recapitulate the in vivo deficiency: a model for rare blood group phenotypes and erythroid membrane disorders. American Journal of Hematology. 88(5), 343-349. doi:10.1002/ajh.23414

Caldwell, L., Harries, P., Sydlik, S., \& Schwiening, C. J. (2013). Presynaptic pH and vesicle fusion in Drosophila larvae neurones. Synapse (New York, N.Y.), 67(11), 729-740. doi: $\underline{10.1002 / s y n .21678}$

Cattaert, D., \& Birman, S. (2001). Blockade of the central generator of locomotor rhythm by noncompetitive NMDA receptor antagonists in Drosophila larvae. Journal of Neurobiology, 48(1), 58-73. 
Chérif-Zahar, B., Raynal, V., Gane, P., Mattei, M. G., Bailly, P., Gibbs, B., ... Cartron, J. P. (1996). Candidate gene acting as a suppressor of the RH locus in most cases of Rhdeficiency. Nature Genetics, 12(2), 168-173. doi:10.1038/ng0296-168

Chérif-Zahar, B., Durand, A., Schmidt, I., Hamdaoui, N., Matic, I., Merrick, M., \& Matassi, G. (2007). Evolution and functional characterization of the RH50 gene from the ammoniaoxidizing bacterium Nitrosomonas europaea. Journal of Bacteriology, 189(24), 9090-9100. doi:10.1128/JB.01089-07

Clark, K. A., Bland, J. M., \& Beckerle, M. C. (2007). The Drosophila muscle LIM protein, Mlp84B, cooperates with D-titin to maintain muscle structural integrity. Journal of Cell Science, 120(12), 2066-2077. doi:10.1242/jcs.000695

Davis, G. W., DiAntonio, A., Petersen, S. A., \& Goodman, C. S. (1998). Postsynaptic PKA controls quantal size and reveals a retrograde signal that regulates presynaptic transmitter release in Drosophila. Neuron, 20(2), 305-315.

Davis, G. W., \& Goodman, C. S. (1998). Synapse-specific control of synaptic efficacy at the terminals of a single neuron. Nature, 392(6671), 82-86. doi10.1038/32176

Durant, A. C., Chasiotis, H., Misyura, L., \& Donini, A. (2017). Aedes aegypti Rhesus glycoproteins contribute to ammonia excretion by larval anal papillae. The Journal of Experimental Biology, 220(Pt 4), 588-596. doi:10.1242/jeb.151084

Durant, A. C., \& Donini, A. (2018). Ammonia excretion in an osmoregulatory syncytium is facilitated by AeAmt2, a novel ammonia transporter in Aedes aegypti larvae. Frontiers in Physiology, 9. doi:10.3389/fphys.2018.00339 
Huang, C.-H., \& Ye, M. (2010). The Rh protein family: gene evolution, membrane biology, and disease association. Cellular and Molecular Life Sciences, 67(8), 1203-1218. doi : $\underline{10.1007 / s 00018-009-0217-x}$

Issa, A.-R., Sun, J., Petitgas, C., Mesquita, A., Dulac, A., Robin, M., ... Birman, S. (2018). The lysosomal membrane protein LAMP2A promotes autophagic flux and prevents SNCAinduced Parkinson disease-like symptoms in the Drosophila brain. Autophagy, 14(11), 1898-1910. doi:10.1080/15548627.2018.1491489

Jan, L. Y., \& Jan, Y. N. (1976). L-glutamate as an excitatory transmitter at the Drosophila larval neuromuscular junction. The Journal of Physiology, 262(1), 215-236. doi:10.1113/jphysiol.1976.sp011593

Jan, L. Y., \& Jan, Y. N. (1982). Antibodies to horseradish peroxidase as specific neuronal markers in Drosophila and in grasshopper embryos. Proceedings of the National Academy of Sciences, 79(8), 2700-2704. doi:10.1073/pnas.79.8.2700

Javelle, A., Severi, E., Thornton, J., \& Merrick, M. (2004). Ammonium sensing in Escherichia coli. Role of the ammonium transporter AmtB and AmtB-GlnK complex formation. The Journal of Biological Chemistry, 279(10), 8530-8538. doi:10.1074/jbc.M312399200

Javelle, A., \& Merrick, M. (2005). Complex formation between AmtB and GlnK: an ancestral role in prokaryotic nitrogen control. Biochemical Society Transactions, 33(1), 170-172. doi: $\underline{10.1042 / B S T 0330170}$

Kirsten, J. H., Xiong, Y., Dunbar, A. J., Rai, M., \& Singleton, C. K. (2005). Ammonium transporter C of Dictyostelium discoideum is required for correct prestalk gene expression 
and for regulating the choice between slug migration and culmination. Developmental Biology, 287(1), 146-156. doi:10.1016/j.ydbio.2005.08.043

Kreipke, R.E., Kwon, Y.V., Shcherbata, H.R., \& Ruohola-Baker, H. (2017). Drosophila melanogaster as a Model of Muscle Degeneration Disorders. Current Topics in Developmental Biology, 121, 83-109. doi:10.1016/bs.ctdb.2016.07.003

LaBeau-DiMenna, E. M., Clark, K. A., Bauman, K. D., Parker, D. S., Cripps, R. M., \& Geisbrecht, E. R. (2012). Thin, a Trim32 ortholog, is essential for myofibril stability and is required for the integrity of the costamere in Drosophila. Proceedings of the National Academy of Sciences of the United States of America, 109(44), 17983-17988. doi: $\underline{10.1073 / p n a s .1208408109}$

Lahey, T., Gorczyca, M., Jia, X., \& Budnik, V. (1994). The Drosophila tumor suppressor gene dlg is required for normal synaptic bouton structure. Neuron, 13(4) 823-835.

Lee, H.-W., Verlander, J. W., Handlogten, M. E., Han, K.-H., \& Weiner, I. D. (2014). Effect of collecting duct-specific deletion of both Rh B Glycoprotein (Rhbg) and Rh C Glycoprotein (Rhcg) on renal response to metabolic acidosis. American Journal of Physiology. Renal Physiology, 306(4), F389-400. doi:10.1152/ajprenal.00176.2013

Lopez, C., Métral, S., Eladari, D., Drevensek, S., Gane, P., Chambrey, R., ... Colin, Y. (2005). The ammonium transporter RhBG: requirement of a tyrosine-based signal and ankyrin-G for basolateral targeting and membrane anchorage in polarized kidney epithelial cells. The Journal of Biological Chemistry, 280(9), 8221-8228. doi:10.1074/jbc.M413351200 
Lorenz, M. C., \& Heitman, J. (1998). The MEP2 ammonium permease regulates pseudohyphal differentiation in Saccharomyces cerevisiae. The EMBO Journal, 17(5), 1236-1247. doi:10.1093/emboj/17.5.1236

Ludewig, U., von Wirén, N., Rentsch, D., \& Frommer, W. B. (2001). Rhesus factors and ammonium: a function in efflux? Genome Biology, 2(3), REVIEWS1010.

Lupo, D., Li, X.-D., Durand, A., Tomizaki, T., Chérif-Zahar, B., Matassi, G., ... Winkler, F. K. (2007). The 1.3-A resolution structure of Nitrosomonas europaea Rh50 and mechanistic implications for $\mathrm{NH}_{3}$ transport by Rhesus family proteins. Proceedings of the National Academy of Sciences of the United States of America, 104(49), 19303-19308. doi: $\underline{10.1073 / p n a s .0706563104}$

Marini, A. M., Matassi, G., Raynal, V., André, B., Cartron, J. P., \& Chérif-Zahar, B. (2000). The human Rhesus-associated RhAG protein and a kidney homologue promote ammonium transport in yeast. Nature Genetics, 26(3), 341-344. doi:10.1038/81656

Marini, A. M., Soussi-Boudekou, S., Vissers, S., \& Andre, B. (1997). A family of ammonium transporters in Saccharomyces cerevisiae. Molecular and Cellular Biology, 17(8), 4282-4293. doi: $10.1128 / \mathrm{mcb} \cdot 17.8 .4282$

Marino, R., Melillo, D., Filippo, M. D., Yamada, A., Pinto, M. R., Santis, R. D., ... Matassi, G. (2007). Ammonium channel expression is essential for brain development and function in the larva of Ciona intestinalis. Journal of Comparative Neurology, 503(1), 135-147. doi $\underline{10.1002 / c n e .21370}$ 
Matassi, G. (2017). Horizontal gene transfer drives the evolution of Rh50 permeases in prokaryotes. BMC Evolutionary Biology, 17, 2. doi:10.1186/s12862-016-0850-6

Marrus, S.B., Portman, S.L., Allen, M.J., Moffat, K.G., \& DiAntonio, A. (2004). Differential localization of glutamate receptor subunits at the Drosophila neuromuscular junction. Journal of Neuroscience, 24(6), 1406-1415. doi:10.1523/JNEUROSCI.1575-03.2004

Menon, K. P., Carrillo, R. A., \& Zinn, K. (2013). Development and plasticity of the Drosophila larval neuromuscular junction. Wiley Interdisciplinary Reviews. Developmental Biology, 2(5), 647-670. doi:10.1002/wdev.108

Menuz, K., Larter, N. K., Park, J., \& Carlson, J. R. (2014). An RNA-seq screen of the Drosophila antenna identifies a transporter necessary for ammonia detection. PLoS Genetics, 10(11), e1004810. doi10.1371/journal.pgen.1004810

Nash, R., \& Shojania, A. M. (1987). Hematological aspect of Rh deficiency syndrome: A case report and a review of the literature. American Journal of Hematology, 24(3), 267-275.

Nicolas, V., Le Van Kim, C., Gane, P., Birkenmeier, C., Cartron, J.-P., Colin, Y., \& MouroChanteloup, I. (2003). Rh-RhAG/ankyrin-R, a new interaction site between the membrane bilayer and the red cell skeleton, is impaired by Rh(null)-associated mutation. The Journal of Biological Chemistry, 278(28), 25526-25533. doi:10.1074/jbc.M302816200

Norenberg, M. D., \& Martinez-Hernandez, A. (1979). Fine structural localization of glutamine synthetase in astrocytes of rat brain. Brain Research, 161(2), 303-310. 
Pitts, R. J., Derryberry, S. L. Jr., Pulous, F. E., \& Zwiebel, L. J. (2014). Antennal-expressed ammonium transporters in the malaria vector mosquito Anopheles gambiae. PLOS ONE, 9(10), e111858. doi:10.1371/journal.pone.0111858

Planelles, G. (2007). Ammonium homeostasis and human Rhesus glycoproteins. Nephron Physiology, 105(1), p11-17. doi:10.1159/000096979

Parnas, D., Haghighi, A.P., Fetter, R.D., Kim, S.W., \& Goodman, C.S. (2001). Regulation of postsynaptic structure and protein localization by the Rho-type guanine nucleotide exchange factor dPix. Neuron, 32(3), 415-424.

Qin, G., Schwarz, T., Kittel, R.J., Schmid, A., Rasse, T.M., Kappei, D., ... Sigrist, S.J. (2005). Four different subunits are essential for expressing the synaptic glutamate receptor at neuromuscular junctions of Drosophila. Journal of Neuroscience, 25(12), 3209-18. doi:10.1523/JNEUROSCI.4194-04.2005

Qiu, J., Tsien, C., Thapalaya, S., Narayanan, A., Weihl, C. C., Ching, J. K., ... Dasarathy, S. (2012). Hyperammonemia-mediated autophagy in skeletal muscle contributes to sarcopenia of cirrhosis. American Journal of Physiology-Endocrinology and Metabolism, 303(8), E983-E993. doi:10.1152/ajpendo.00183.2012

Riemensperger, T., Issa, A.R., Pech, U., Coulom, H., Nguyễn, M.V., Cassar, M., ... Birman, S. (2013). A single dopamine pathway underlies progressive locomotor deficits in a Drosophila model of Parkinson disease. Cell Reports 5(4), 952-960. doi:10.1016/j.celrep.2013.10.032 
Ripoche, P., Bertrand, O., Gane, P., Birkenmeier, C., Colin, Y., \& Cartron, J.P. (2004). Human Rhesus-associated glycoprotein mediates facilitated transport of $\mathrm{NH}(3)$ into red blood cells. Proc Natl Acad Sci U S A, 101(49):17222-17227. doi:10.1073/pnas.0403704101

Schindelin, J., Arganda-Carreras, I., Frise, E., Kaynig, V., Longair, M., Pietzsch, T., ... Cardona, A. (2012). Fiji: an open-source platform for biological-image analysis. Nature Methods, 9(7), 676-682. doi:10.1038/nmeth.2019

Stewart, B. A., Schuster, C. M., Goodman, C. S., \& Atwood, H. L. (1996). Homeostasis of synaptic transmission in Drosophila with genetically altered nerve terminal morphology. Journal of Neuroscience, 16(12), 3877-3886.

Strubberg, A. M., Liu, J., Walker, N. M., Stefanski, C. D., MacLeod, R. J., Magness, S. T., \& Clarke, L. L. (2018). Cftr Modulates Wnt/ß-Catenin Signaling and Stem Cell Proliferation in Murine Intestine. Cellular and Molecular Gastroenterology and Hepatology, 5(3), 253-271. doi:10.1016/j.jcmgh.2017.11.013

The FlyBase Consortium. (2003). The FlyBase database of the Drosophila genome projects and community literature. Nucleic Acids Research, 31(1), 172-175.

von Wirén, N., \& Merrick, M. (2004). Regulation and function of ammonium carriers in bacteria, fungi, and plants. In Molecular Mechanisms Controlling Transmembrane Transport (p. 95-120). Springer, Berlin, Heidelberg.

Wagh, D.A., Rasse, T.M., Asan, E., Hofbauer, A., Schwenkert, I., Dürrbeck, H., ...Buchner, E. (2006). Bruchpilot, a protein with homology to ELKS/CAST, is required for structural integrity and function of synaptic active zones in Drosophila. Neuron 49(6), 833-844. 
doi:10.1016/j.neuron.2006.02.008

Weihrauch, D. (2006). Active ammonia absorption in the midgut of the Tobacco hornworm Manduca sexta L.: transport studies and mRNA expression analysis of a Rhesus-like ammonia transporter. Insect Biochemistry and Molecular Biology, 36(10), 808-821. doi:10.1016/j.ibmb.2006.08.002

Weiner, I. D., \& Verlander, J. W. (2017). Ammonia transporters and their role in acid-base balance. Physiological Reviews, 97(2), 465-494. doi:10.1152/physrev.00011.2016

Wright, P. A., \& Wood, C. M. (2009). A new paradigm for ammonia excretion in aquatic animals: role of Rhesus (Rh) glycoproteins. The Journal of Experimental Biology, 212(Pt 15), 2303-2312. doi: $10.1242 / \mathrm{jeb} .023085$

Wu, Y., Zheng, X., Zhang, M., He, A., Li, Z., \& Zhan, X. (2010). Cloning and functional expression of Rh50-like glycoprotein, a putative ammonia channel, in Aedes albopictus mosquitoes. Journal of Insect Physiology, 56(11), 1599-1610.

doi:10.1016/j.jinsphys.2010.05.021

Zhao, J., Brault, J. J., Schild, A., Cao, P., Sandri, M., Schiaffino, S., ... Goldberg, A. L. (2007). FoxO3 coordinately activates protein degradation by the autophagic/lysosomal and proteasomal pathways in atrophying muscle cells. Cell Metabolism, 6(6), 472-483. doi:10.1016/j.cmet.2007.11.004 
TABLE 1 Primers used for PCR and real-time PCR

\begin{tabular}{|c|c|}
\hline Primers $\dagger$ & Sequences $\dagger^{+}$ \\
\hline $\mathrm{p} 1:$ Rh50-EcoRI (f) & 5’-CCGCGAATTCATCTTGCCACCATGCATTCG-3’ \\
\hline p2 : Rh50A-BglII (r) & 5’-GTCACTAGAGATCTAGTTCCTATTCCTC-3’’ \\
\hline p3 : pT7-RhCG-BglII (f) & 5’- GTCCATAGATCTCAGATATCTAGCATGGCCTGG-3’ \\
\hline p4 : pT7-RhCG-XhoI (r) & 5’-CACCTCGAGCTCСТCACCTGCCCTGGGAGCCTAGGG-3’ \\
\hline p5 : Rh50 (f) & 5’-TTCCTCCACATGGAGGGCGGCAAG-3’' \\
\hline p6 : Rh50 (r) & 5'-GCGGATACAACGAAGGTGGTCACTG-3' \\
\hline $\mathrm{p} 7: \operatorname{Rh} 50(\mathrm{f})$ & 5’-CTCGGTGCTCGGCTATCGCTATTTAACAC-3’ \\
\hline p8 : Rh50A (r) & 5’-GTGATCAATCTGACAGGCACAACACTTAAC-3’ \\
\hline p9 : Rh50BC (r) & 5’-TCGTCAACTTTTTCTAGTTCATATTCC-3’ \\
\hline $\mathrm{p} 10: \operatorname{Rp} 49(\mathrm{f})$ & 5’-GACGCTTCAAGGGACAGTATC-3’ \\
\hline $\mathrm{p} 11: \operatorname{Rp} 49(\mathrm{r})$ & 5’-AAACGCGGTTCTGCATGAG-3’ \\
\hline
\end{tabular}

$\dagger(\mathrm{f})$ : forward ; $(\mathrm{r})$ : reverse. $\dagger$ The added restriction sites are in bold type. 
TABLE 2 Antibodies used in this study

\begin{tabular}{|c|c|c|c|c|}
\hline Antibody & Immunogen $\dagger$ & $\begin{array}{c}\text { Final } \\
\text { concent. }\end{array}$ & $\begin{array}{c}\text { Host } \\
\text { species }\end{array}$ & Source $† \dagger$ \\
\hline Rh50 & $\begin{array}{l}\text { Drosophila aa } 43-60 \\
\text { and } 423-440\end{array}$ & $1: 250$ & Rabbit & Eurogentec - This work \\
\hline GluRIIA & Drosophila aa 633-786 & $1: 50$ & Mouse & $\begin{array}{l}\text { DSHB - Depositor C. Goodman } \\
\text { RRID : AB_528269 }\end{array}$ \\
\hline GluRIIB & $\begin{array}{l}\text { Drosophila C-terminal } \\
\text { aа } 899-913\end{array}$ & $1: 1000$ & Rabbit & $\begin{array}{l}\text { Y. Grosjean } \\
\text { RRID :AB_2568753 }\end{array}$ \\
\hline GluRIID & $\begin{array}{l}\text { Drosophila C-terminal } \\
\text { aa } 888-902\end{array}$ & $1: 500$ & Rabbit & SJ. Sigrist, RRID :AB_2569238 \\
\hline Dlg & $\begin{array}{l}\text { Drosophila PDZ2 } \\
\text { domain aa 439-756 }\end{array}$ & $1: 500$ & Mouse & $\begin{array}{l}\text { DSHB - Depositor C. Goodman } \\
\text { RRID : AB } 528203\end{array}$ \\
\hline Brp (NC82) & $\begin{array}{l}\text { Drosophila C-terminal } \\
\text { aа } 1227-1740\end{array}$ & $1: 500$ & Mouse & $\begin{array}{l}\text { DSHB - Depositor E. Buchner } \\
\text { RRID : AB_2314866 }\end{array}$ \\
\hline GFP & $\begin{array}{l}\text { Aequoria victoria } \\
\text { Recombinant wt } \\
\text { protein }\end{array}$ & $1: 250$ & Mouse & $\begin{array}{l}\text { DSHB - Depositor DSHB } \\
\text { GFP-12A6 }\end{array}$ \\
\hline HRP-FITC & $\begin{array}{l}\text { Peroxydase from the } \\
\text { horseradish }\end{array}$ & $1: 10$ & Goat & $\begin{array}{l}\text { Jackson ImmunoResearch } \\
\text { RRID:AB_2314647 }\end{array}$ \\
\hline $\begin{array}{l}\text { AlexaFluor } \\
\quad 488 \\
\text { (anti-mouse) }\end{array}$ & Molecular Probes & $1: 1000$ & Goat & RRID: AB_2534088 \\
\hline $\begin{array}{l}\text { AlexaFluor } \\
555 \\
\text { (anti-rabbit) }\end{array}$ & Molecular Probes & $1: 1000$ & Goat & RRID: AB_2535849 \\
\hline
\end{tabular}

† aa: amino acids; wt: wild type. †† DSHB: Developmental Studies Hybridoma Bank 


\section{Figure legends}

Figure 1. Relative abundance of Drosophila $R$ h50 transcripts and localization of the protein in muscles and NMJs of third-instar larvae. (a) Three mRNAs, $R h 50 A, R h 50 B$ and $R h 50 C$ are produced through alternative splicing of the $R h 50$ primary transcript. The last nucleotide (nt) of the $R h 50 A$ stop codon is eliminated together with 237 nt from the 3'-untranslated region in Rh50B and Rh50C mRNAs, adding 11 supplementary codons and more polyadenylation sites. The double-stranded interfering Rh50 RNA from the UAS-iRh construct encompasses $300 \mathrm{nt}$ overlapping exon 4 and 5 (red lines). (b) RT-qPCR analysis of Rh50 mRNA abundance in whole third-instar larvae. The primers used to amplify Rh50B and Rh50C were common to these two isoforms. $R h 50 A$ mRNAs were quantified using $R p 49$ as a reference and the $R h 50 B / R h 50 C$ mRNA level was compared to that of Rh50A mRNA. (c) Confocal projections of third-instar larval NMJs on ventral longitudinal muscles 6 and 7, stained with anti-HRP (green), a neuronal membrane marker, and anti-Rh50 (magenta) antibodies. Rh50 is expressed in muscles and strongly enriched at the NMJs. Scale bar: $30 \mu \mathrm{m}$. (d) Rh50 immunostaining surrounds the fluorescence of presynaptic msGFP expressed in motor neurons with elav-Gal4. Scale bar: 10 $\mu m$. (e) Wild-type larva NMJ of muscles 6 and 7 labeled with anti-Rh50 (magenta) and anti-Dlg (green), a postsynaptic marker. Rh50 and Dlg co-localize around the periphery of synaptic boutons, as confirmed by the yz confocal scan (right). Scale bar: $5 \mu \mathrm{m}$. (f) Confocal Z-projection

of third instar larval NMJs stained with the Rh50 antibody. Overexpression of DmRh50A cDNA 
with the muscle driver 24B-Gal4 (24B>Rh50) increased Rh50 immunofluorescence intensity in the NMJs compared to its normal level of expression in control wild-type larvae.

Figure 2. Rh50 inactivation induces muscular atrophy and pupal lethality. (a) Rh50-deficient pupae $\left(d a>R h 50^{\mathrm{RNAi}}\right)$ are thinner and longer than wild-type pupae $\left(w^{1118}\right)$ and they never give rise to adults. Scale bar: $1 \mathrm{~mm}$. (b) Immunofluorescence staining of body wall muscles of third-instar larvae using anti-Dlg antibody. Rh50 inactivation in muscles $\left(24 B>R h 50^{\mathrm{RNAi}}\right)$ resulted in thinner muscles, with an unstructured shape. Muscles 6 and 7 of A3 segments used for muscle size quantification are indicated by the white box. Scale bar: $200 \mu \mathrm{m}$. (c) Quantification of the width of larval muscles 6 and 7. Compared to the size of muscles from control larvae $(24 B /+)$, the muscle width in Rh50-deficient larvae (24B $>R h 50^{\mathrm{RNAi}}$ ) was significantly smaller (respectively, $169.9 \mu \mathrm{m} \pm 5.0 v s 134.5 \mu \mathrm{m} \pm 2.8,{ }^{* *} p<0.01$ ). Expression of human $R h C G$ rescued the width of the body wall muscles in Rh50-deficient larvae (24B>Rh50 ${ }^{\mathrm{RNAi}}$, RhCG: $152.4 \mu \mathrm{m} \pm 2.4$ vs 134.5 $\mu \mathrm{m} \pm 2.8$, \#p <0.05), which were not different anymore from those of the control larvae (ns) ( $\mathrm{N}$ $=4$ independent experiments). (d) Assessment of larval survival by measuring the rate of pupa formation. Rh50-deficient larvae exhibited a decreased rate of pupa formation compared to controls $\left(24 B>R h 50^{\mathrm{RNAi}}: 0.53 \pm 0.09\right.$ vs $\left.24 B /+: 0.91 \pm 0.1,{ }^{* * *} p<0.001\right)$. Expression of human $R h C G$ also partially rescued larval survival $\left(24 B>R h 50^{\mathrm{RNAi}}\right.$, RhCG: $\left.0.67 \pm 0.05, \# p<0.05\right)(\mathrm{N}=$ 7 independent experiments). 
Figure 3. Silencing of $\boldsymbol{R h} 50$ increases GluRIIA expression and does not alter the morphology of the NMJ. (a, b) Quantification of the staining area of the postsynaptic Dlg (a) and the presynaptic Brp (b) markers at the NMJ of larval muscles 6 and 7. Rh50-deficient larvae $\left(24 B>R h 50^{\mathrm{RNAi}}\right)$ exhibited similar Dlg and Brp immunostaining areas as the controls $(24 B /+$ and $\left.R h 50^{\mathrm{RNAi}} /+\right)$. Relatively to the control group $24 \mathrm{~B} /+$, Dlg: $\left(24 B>R h 50^{\mathrm{RNAi}} 0.90 \pm 0.06\right.$ and $\left.R h 50^{\mathrm{RNAi}} /+0.86 \pm 0.05\right)$; $\mathrm{Brp}:\left(24 B>R h 50^{\mathrm{RNAi}} 0.91 \pm 0.02\right.$ and $\left.R h 50^{\mathrm{RNAi}} /+1.06 \pm 0.10\right)(\mathrm{N}=1)$. The quantification was performed on the NMJ of 4 larvae per genotype. (c, d) Quantification of the number of type Ib (c) and type Is (d) synaptic boutons at the NMJ of muscles 6 and 7. Rh50deficient larvae $\left(24 B>R h 50^{\mathrm{RNAi}}\right)$ exhibit comparable number of type Ib and Is boutons with controls (24B/+ and $\left.R h 50^{\mathrm{RNAi}} /+\right)$. Ib: $\left(24 B>R h 50^{\mathrm{RNAi}}: 30 \pm 0, R h 50^{\mathrm{RNAi}} /+: 36 \pm 7,24 B /+: 22 \pm 5\right)$; Is: $\left(24 B>R h 50^{\mathrm{RNAi}} 25 \pm 2, R h 50^{\mathrm{RNAi}} /+\right.$ : $\left.33 \pm 1,24 B /+: 26 \pm 6\right)(\mathrm{N}=2$ independent experiments, $\mathrm{n}$ $\geq 5$ larvae). (e) Anti-GluRIIA immunostaining indicates that expression of glutamate receptor Asubunit is increased in muscles of Rh50-deficient larvae $\left(24 B>R h 50^{\mathrm{RNAi}}\right)$ compared to wild-type larvae (24B/+). Scale bar: $130 \mu \mathrm{m}$. (f) Western blot of larval carcass extracts (body wall muscles and cuticle) prepared from control $\left(24 B /+, R h 50^{\mathrm{RNAi}} /+\right)$ and $R h 50$-deficient $\left(24 B>R h 50^{\mathrm{RNAi}}\right)$ larvae probed with anti-GluRIIA and anti- $\alpha$-tubulin antibodies. GluRIIA expression is strongly increased in Rh50 deficiency conditions. (g) Quantification of relative GluRIIA immunofluorescence intensity in extrasynaptic regions of the muscles (relatively to the control group, $24 B>R h 50^{\mathrm{RNAi}}: 1.536 \pm 0.052, R h 50^{\mathrm{RNAi}} /+: 1.015 \pm 0.060$; ANOVA test, $\mathrm{p}=0.0003$; N $=4$ independent experiments) and at the NMJ (relatively to the control group 24B/+, 
$24 B>R h 50^{\mathrm{RNAi}}: 1.184 \pm 0.140, R h 50^{\mathrm{RNAi}} /+: 1.126 \pm 0.070 ; \mathrm{N}=6$ independent experiments).

(h) Quantification of GluRIIA by western blot normalized to alpha-tubulin abundance: compared to the control group $R h 50^{\mathrm{RNAi}} /+, 24 B>R h 50^{\mathrm{RNAi}}: 8.20 \pm 1.21,24 B /+: 1.14 \pm 0.22$; ANOVA test, $p=0.0006)$.

Figure 4. Rh50 is required to maintain GluRIIA expression at attachment sites. (a-c) Confocal projections of third-instar larval muscles stained with anti-GluRIIA antibody. (a) In wild type $(24 B /+)$ GluRIIA immunoreactivity is concentrated at attachment sites in lateral muscles (arrows) and in muscle-muscle junction (asterisks). (b) When Rh50 is knocked down (24B $>$ Rh50 ${ }^{\mathrm{RNAi}}$ ) GluRIIA expression is no longer constrained to the muscle attachment sites (arrows and asterisks) but highly expressed and dispersed in the muscle. (c) In 24B $>$ thin $^{\text {RNAi }}$ GluRIIA expression at attachment sites is less structured than wild type. Scale bar: $160 \mu \mathrm{m}$.

\section{Figure 5. Rh50 inactivation in muscles decreases larval locomotor performance. (a)}

Schematic of the successive phases of larval locomotion. The locomotor stride is defined as the distance crawled during one peristaltic wave of muscle contraction. (b) Compared to the controls, the locomotor stride of third-instar larvae was reduced in $R h 50$-deficient larvae $\left(24 \mathrm{~B}>R h 50^{\mathrm{RNAi}}\right)$ : (in mm/peristaltic wave, $24 B>R h 50^{\mathrm{RNAi}}$ : $0.529 \pm 0.035 ; 24 \mathrm{~B} /+: 0.843 \pm 0.048 ; R h 50^{\mathrm{RNAi}} /+$ : 0.875 $\left.\pm 0.021 ;{ }^{* * *} p=0.0002\right)(\mathrm{N}=4$ independent experiments). (c) Inactivating $\mathrm{Rh} 50$ in neurons did not affect the locomotor stride (in mm/peristaltic wave, elav $>$ Rh50 ${ }^{\mathrm{RNAi}}$ : $0.81 \pm 0.04$; elav/+: 0.74 
\pm 0.03 ), neither did inactivation in glia (in mm/peristaltic wave, repo $>R h 50^{\mathrm{RNAi}}: 0.68 \pm 0.04$; repo/+: $0.59 \pm 0.05) \mathrm{n} \geq 5$ larvae.

Figure 6. Inactivating $\boldsymbol{R h} 50$ in muscles increases miniature EPSC frequency. Spontaneous NMJ activity assessed by electrophysiological recording of muscles 6 and 7 of third-instar $R h 50$ deficient $\left(24 \mathrm{~B}>R h 50^{\mathrm{RNAi}}\right)$ and control $\left(24 \mathrm{~B} /+\right.$ and $\left.R h 50^{\mathrm{RNAi}} /+\right)$ larvae. Representative voltage clamp recordings of NMJ spontaneous activity of control $\left(R h 50^{\mathrm{RNAi}} /+\right)$ (a) and $R h 50^{\mathrm{RNAi}}$ (b) larvae. (c) The mean amplitude of miniature excitatory currents (mEPSCs) recorded in voltage clamp was not significantly different from controls $\left(24 \mathrm{~B} /+\right.$ and $\left.R h 50^{\mathrm{RNAi}} /+\right)$ and $R h 50^{\mathrm{RNAi}}$ larvae $(-0.81 \pm 0.08 \mathrm{nA} v s-0.67 \pm 0.06 \mathrm{nA})$. (d) In contrast, the mean instantaneous frequency of miniature excitatory potentials (mEPSPs) recorded in current clamp was increased in $R h 50^{\mathrm{RNAi}}$ compared to control larvae $\left(4.67 \mathrm{~Hz} \pm 0.50\right.$ vs $\left.3.05 \mathrm{~Hz} \pm 0.36,{ }^{*} p=0.020\right)$. In c and d, the numbers below the graphs indicate the number of larvae tested in each condition. 
The Drosophila melanogaster Rh50 protein is expressed in larval muscle and enriched at the neuromuscular junction. Rh50 inactivation by RNAi selectively in muscle cells caused muscular atrophy, locomotor defect, increase of GluRIIA expression and pupal lethality. 

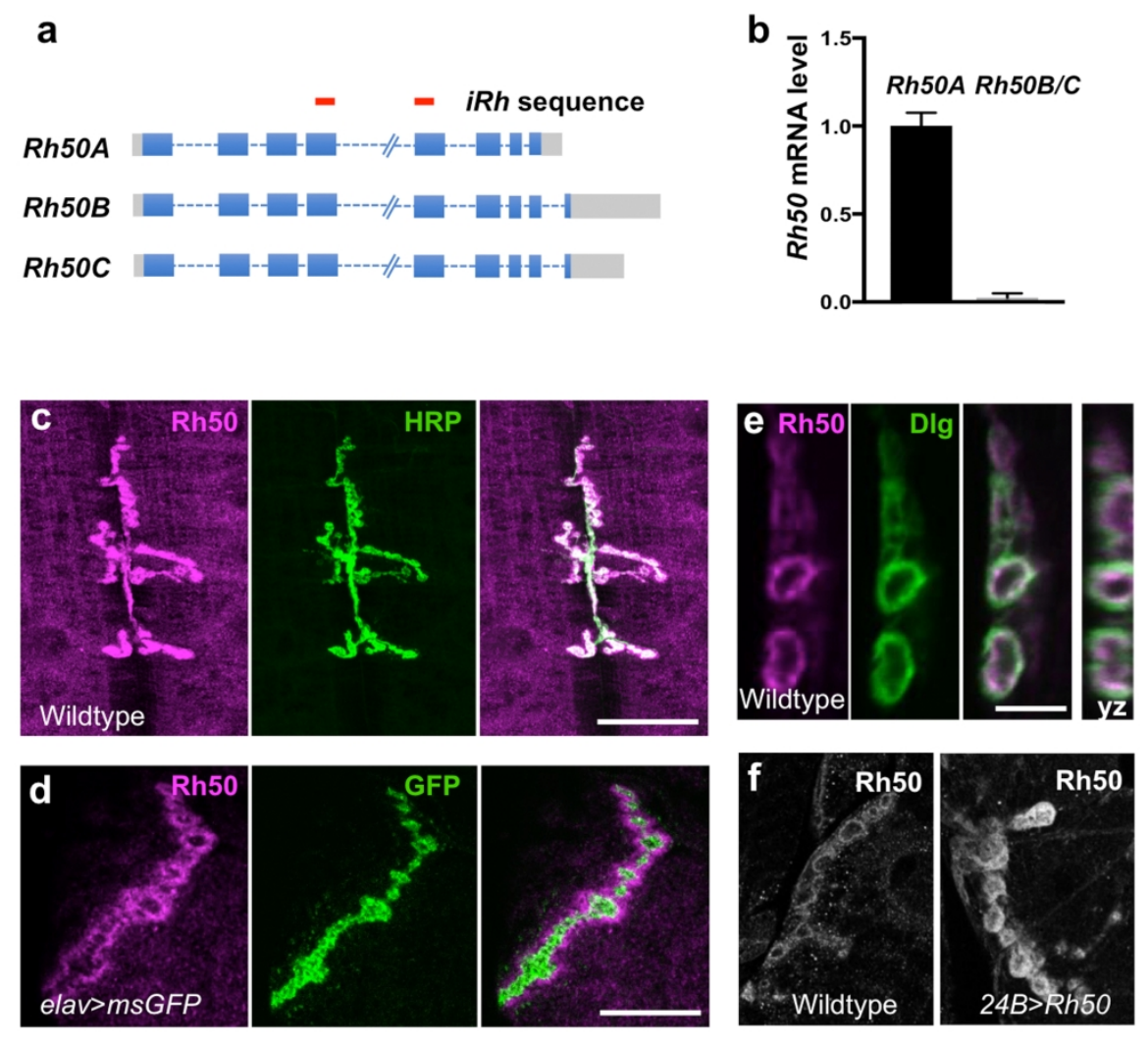

Figure 1 
a
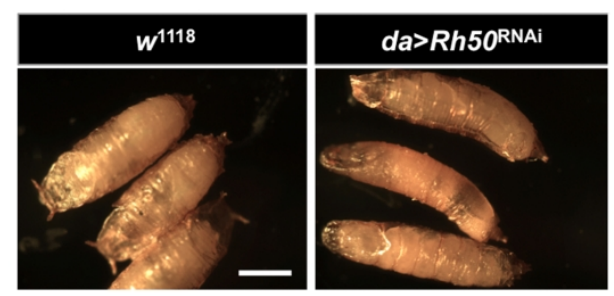

b

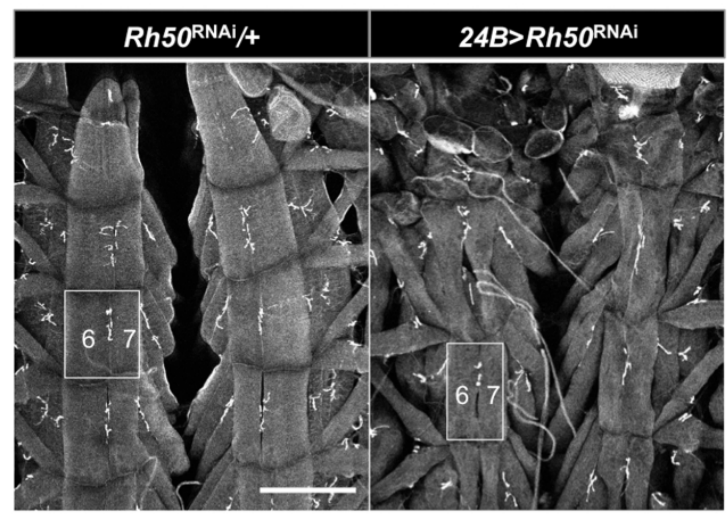

C

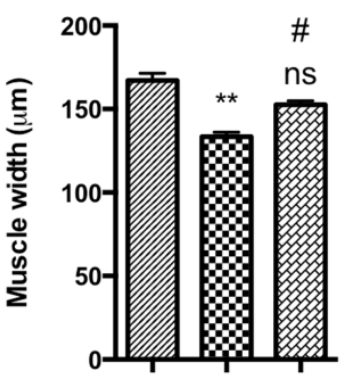

$24 B /+$

d $\mathbf{W} 24 B>R h 50^{R N A i}$ 24B $>$ Rh50 RNAi, $R h C G$

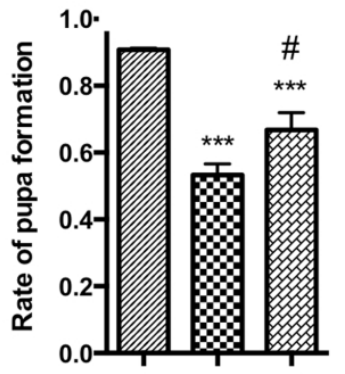

Figure 2 

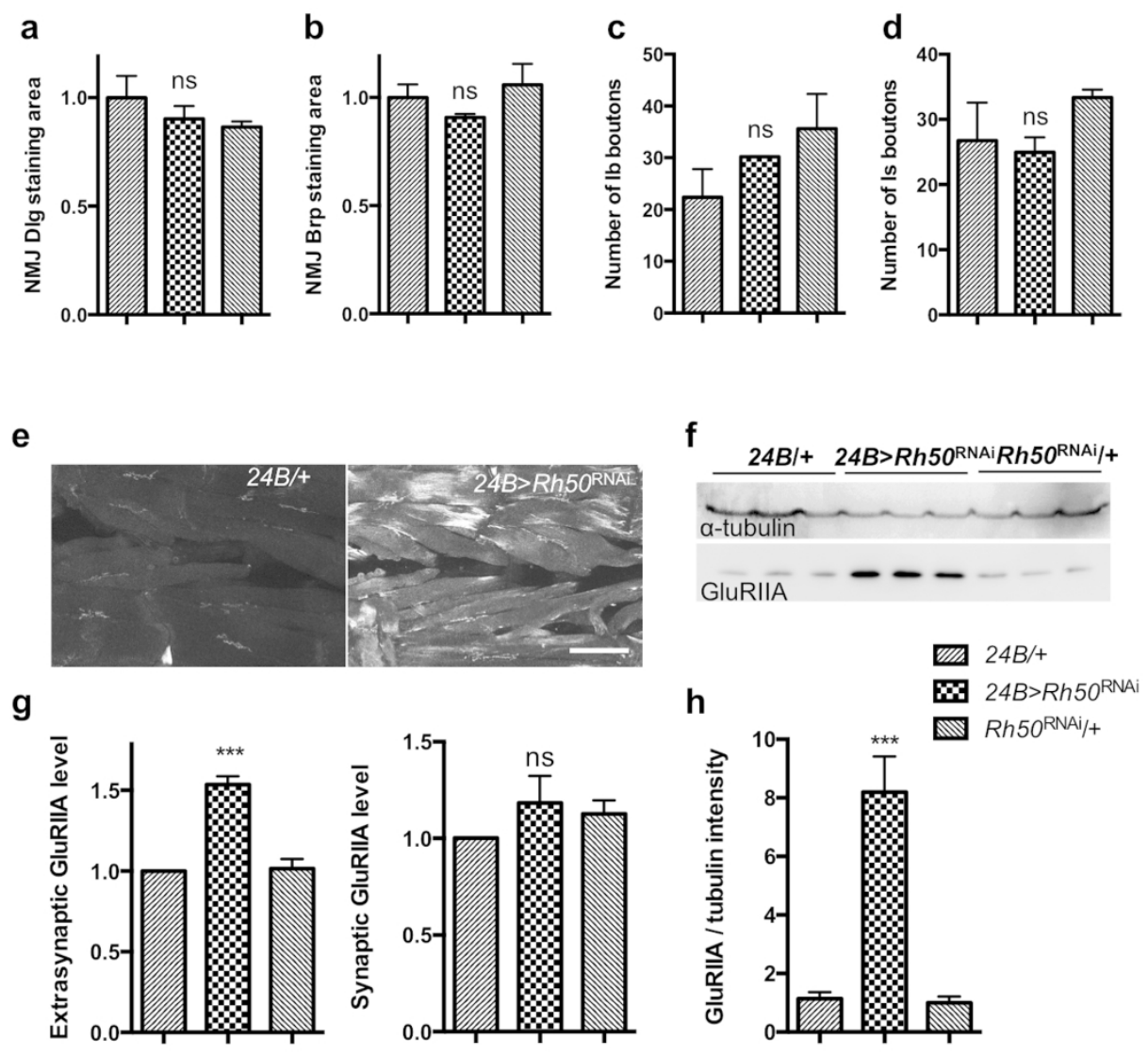

Figure 3 

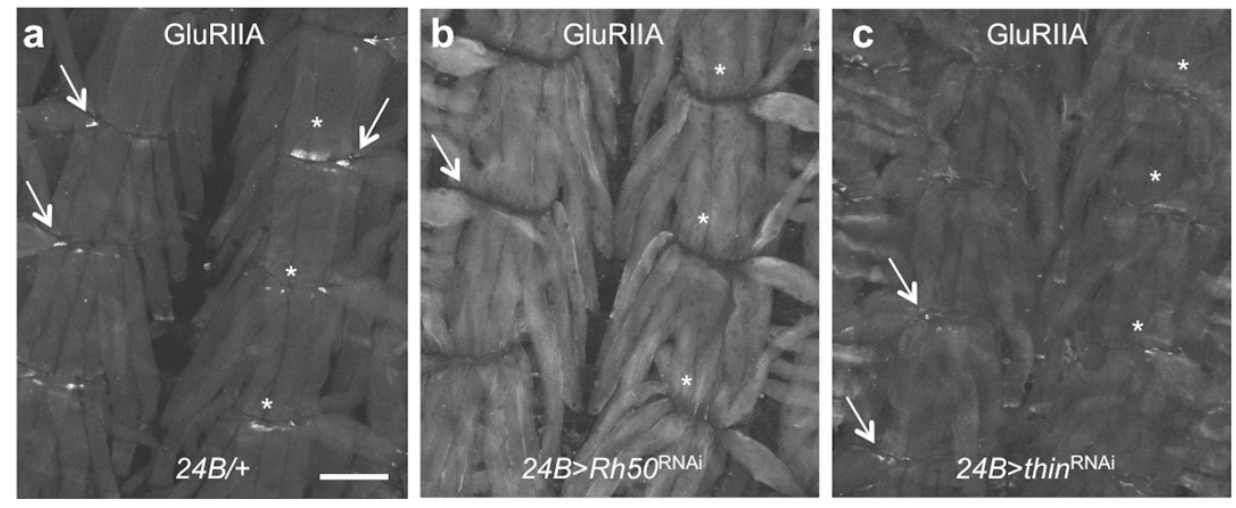

Figure 4 

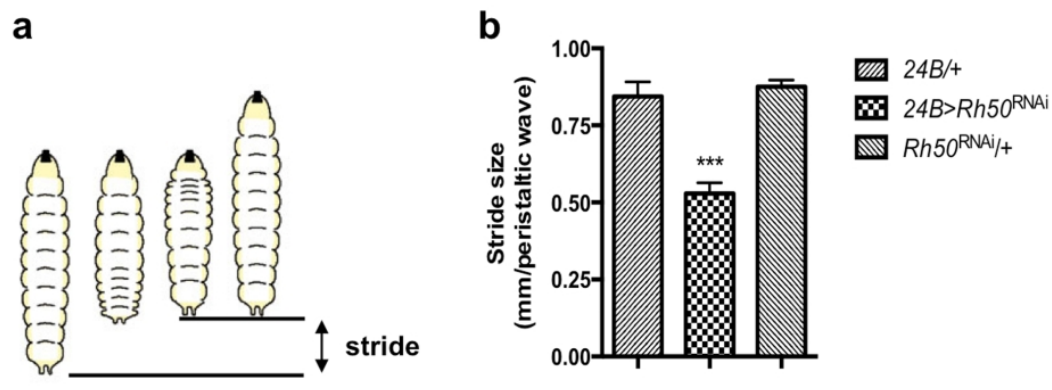

C

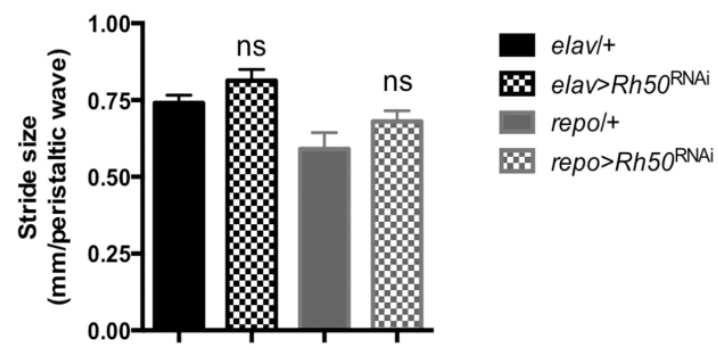

Figure 5 

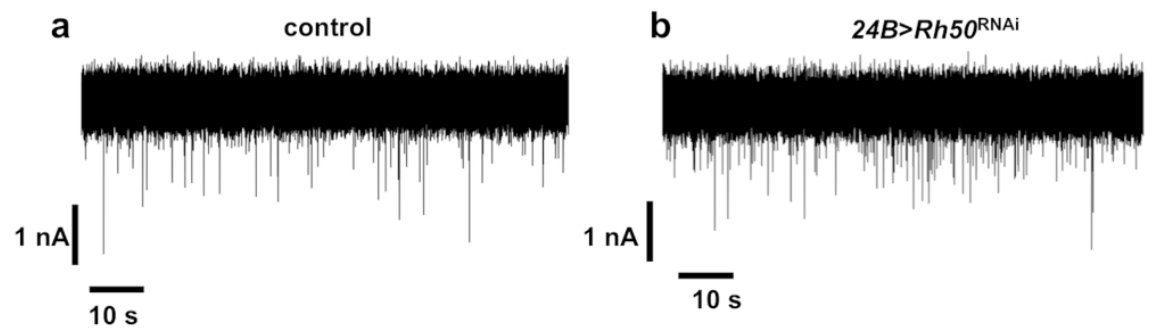

C

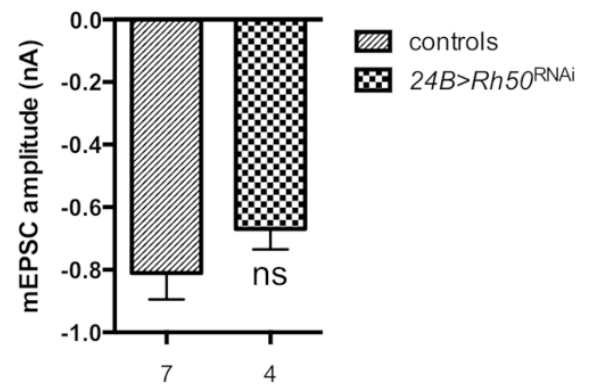

d

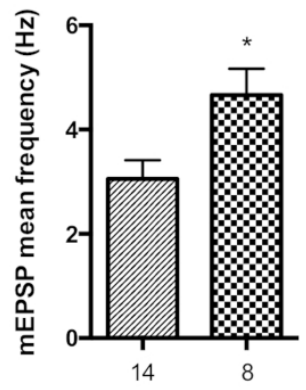

Figure 6 